\title{
The Zanstra Temperatures Revisited
}

\author{
R. Gruenwald and S. M. Viegas \\ Instituto de Astronomia, Geofisica e Ciências Atmosféricas. São Paulo, \\ Brazil
}

The temperature of the central stars in planetary nebulae is generally obtained by the Zanstra method. This method provides two values for the temperature of a single star: one obtained from the $\mathrm{H}$ recombination lines, $\mathrm{T}_{Z}(\mathrm{H})$, and another from the $\mathrm{He}$ recombination lines, $\mathrm{T}_{Z}(\mathrm{He}$ II). The ratio (ZR) of these two values can be different from unit, leading to the well-known "Zanstra discrepancy". The discrepancy is higher for lower temperature stars and the method does not reproduce the high values of stellar temperatures suggested by stellar evolutionary models. In this work a careful analysis of the effect of the optical depth on the determination of the Zanstra temperature is made, using photoionization models. The effects due to deviations from a blackbody spectrum, as well as to the He abundance in the nebulae, are also discussed. We show, quantitatively, that the details of the distribution of the $\mathrm{H}$ and $\mathrm{He}$ II Zanstra temperatures are mainly explained by an optical depth effect; in particular, the fact that the discrepancy is larger for low stellar temperatures. The results also show that for high stellar temperatures both Zanstra temperatures underestimate the stellar temperature, even for high optical depths. The stellar temperature, as well as the optical depth, can be obtained from a plot of ZR vs. $\mathrm{T}_{Z}(\mathrm{He}$ II). Since for nebulae of very low optical depth and/or high stellar temperature this plot only provides lower limits for $\mathrm{T}_{*}$, we propose the use of the line intensity ratio $\mathrm{He}$ $\mathrm{II} / \mathrm{He} \mathrm{I}$ vs. $\mathrm{T}_{Z}(\mathrm{He} \mathrm{II})$ diagram for obtaining the stellar temperature, as well as the nebular optical depth. The results of this work has been published in ApJ 543,889 (2000).

Acknowledgments. This work was partially supported by Fapesp and Pronex/Finep 